Journal for ImmunoTherapy of Cancer

\section{Nivolumab-associated DRESS in a genetic susceptible individual}

To cite: Ai L, Gao J, Zhao S, et al. Nivolumab-associated DRESS in a genetic susceptible individual. Journal for ImmunoTherapy of Cancer 2021;9:e002879. doi:10.1136/ jitc-2021-002879

LA, JG and SZ contributed equally.

Accepted 16 July 2021

\section{Check for updates}

(c) Author(s) (or their employer(s)) 2021. Re-use permitted under CC BY-NC. No commercial re-use. See rights and permissions. Published by BMJ.

${ }^{1}$ Department of Medical Oncology, Zhongshan Hospital Fudan University, Shanghai, China

${ }^{2}$ Cancer Center, Zhongshan Hospital Fudan University,

Shanghai, China

${ }^{3}$ Department of Radiology, Zhongshan Hospital Fudan University, Shanghai, China ${ }^{4}$ Department of Nephrology, Zhongshan Hospital Fudan University, Shanghai, China ${ }^{5}$ Department of Pathology, Zhongshan Hospital Fudan University, Shanghai, China ${ }^{6}$ Genecast Biotechnology Co Ltd, Wuxi City, China

Correspondence to

Dr Tianshu Liu;

liu.tianshu@zs-hospital.sh.cn

Yiyi Yu; Yu.yiyi@zs-hospitsl.sh.cn

\section{ABSTRACT}

The use of immune checkpoint inhibitors (ICls) is rising exponentially in numerous cancers, but immune-related adverse events can occur. We report a rare case of high-grade drug reaction with eosinophilia and systemic symptoms (DRESS) syndrome developed stepwise in a patient with gastric cancer after nivolumab treatment. Subclinical myocarditis was sensitively detected by cardiovascular magnetic resonance 3 weeks after initiating nivolumab. Eruption, eosinophilia, and interstitial pneumonitis occurred 1 week later. Corticosteroids were started and his condition improved. Four months later, when he was still on steroids tapering off, acute kidney injury and sequential herpes zoster virus activation developed. Severe acute tubulointerstitial nephritis (ATN) with an intense infiltration of lymphocytes was observed on renal biopsy. In blood, a substantial shift to Th2 response, an increase of Th17 cells, and strikingly enriched granzyme $\mathrm{B}^{+}$and perforin ${ }^{+} \mathrm{CD}^{+} \mathrm{T}$ cells were detected at ATN onset. Serum interleukin (IL)-5, IL-17, interferon gamma, and IL-6 levels were consistently elevated. Further molecular profiling identified a DRESS risk allele human leukocyte antigen (HLA)- $A^{\star} 31: 01$ in this patient. His ATN responded favorably to a high dose of corticosteroids. In parallel, complete antitumor response was observed during the clinical course of DRESS. This is the first ever case report of nivolumab-associated DRESS syndrome with exploration of the mechanisms from the histopathological, cellular and molecular aspects. Nivolumab-induced DRESS may result from type IV hypersensitivity-related 'off-target effect' and PD-1 blockmediated 'on-target effect'. HLA risk alleles may constitute the genetic susceptible basis. HLA typing assay has the potential to screen susceptible individuals to avoid ICIinduced DRESS.

\section{INTRODUCTION}

Immune checkpoint inhibitors (ICIs) have transformed the treatment of multiple cancers by normalization of restrained antitumor immune responses. ${ }^{1}$ Alongside its remarkable success, a diverse and sometimes unpredictable number of inflammatory side effects, collectively referred to as immune-related adverse events (irAEs), may come at a cost. Patients who developed irAEs usually had better antitumor response. ${ }^{23}$ The beneficial antitumor activity and associated development of irAEs appear to share a common origin: both of them are consistent with an overall, rather than selective, enhancement of immune attack. ${ }^{4}$ The proposed immunopathogenic mechanisms of irAEs include, but are not limited to, crossantigen reactivity between tumor cells and host tissue, cytokine/chemokine production, breach of self-tolerance leading to autoantigen reactivity and production of pathogenic autoantibodies, off-target effects of ICIs on other cell lines bearing the target immune checkpoint ligand, and enrichment of certain microbiomes. ${ }^{5}$

Dermatological toxicities are the most frequent irAEs associated with ICI therapy, ${ }^{6}$ with the reported incidence of all grade ranges from $17 \%$ to $40 \%$ for PD-1/PD-L1 inhibitors and from $37 \%$ to $70 \%$ for ipilimumab. Highgrade dermatological adverse events were rare with the reported rates between $1 \%$ and $3 \% .^{7-9}$ Drug reaction with eosinophilia and systemic symptoms (DRESS) is a rare, idiosyncratic but potentially life-threatening adverse drug reaction. It is fewer reported in current literature as a rare side effect of ICIs. Until now, only three cases of ICI-associated DRESS were reported, with nivolumab and/or ipilimumab being the inciting drugs. ${ }^{10-12}$ Common features of DRESS are fever, eosinophilia, lymphocyte activation, multiorgan involvement, and reactivation of herpes viruses, especially HHV6. ${ }^{13}$ Patients usually develop two or three features of symptoms followed by a stepwise development of other symptoms. Here, we report a case of DRESS induced by nivolumab and explored the potential mechanisms from the histopathological, cellular and molecular aspects.

\section{CASE PRESENTATION}

A 72-year-old Chinese man of Han nationality was diagnosed with stage IIIA lowdifferentiated gastric adenocarcinoma and underwent radical distal gastrectomy in 
Baseline

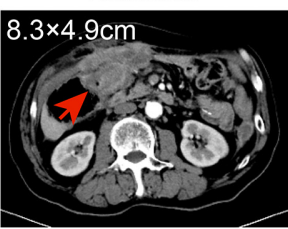

$03 / 25 / 2020$ 2.3months

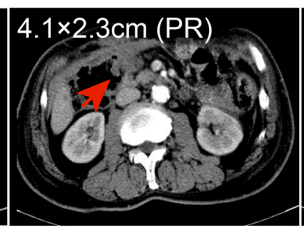

$06 / 03 / 2020$ 5.6months

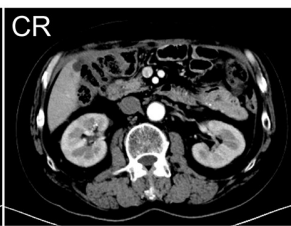

09/14/2020

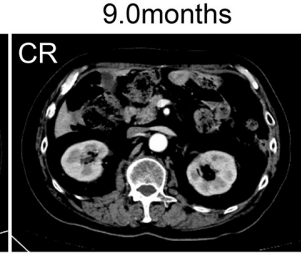

$12 / 28 / 2020$ 13.7months

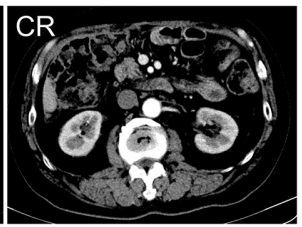

05/19/2021

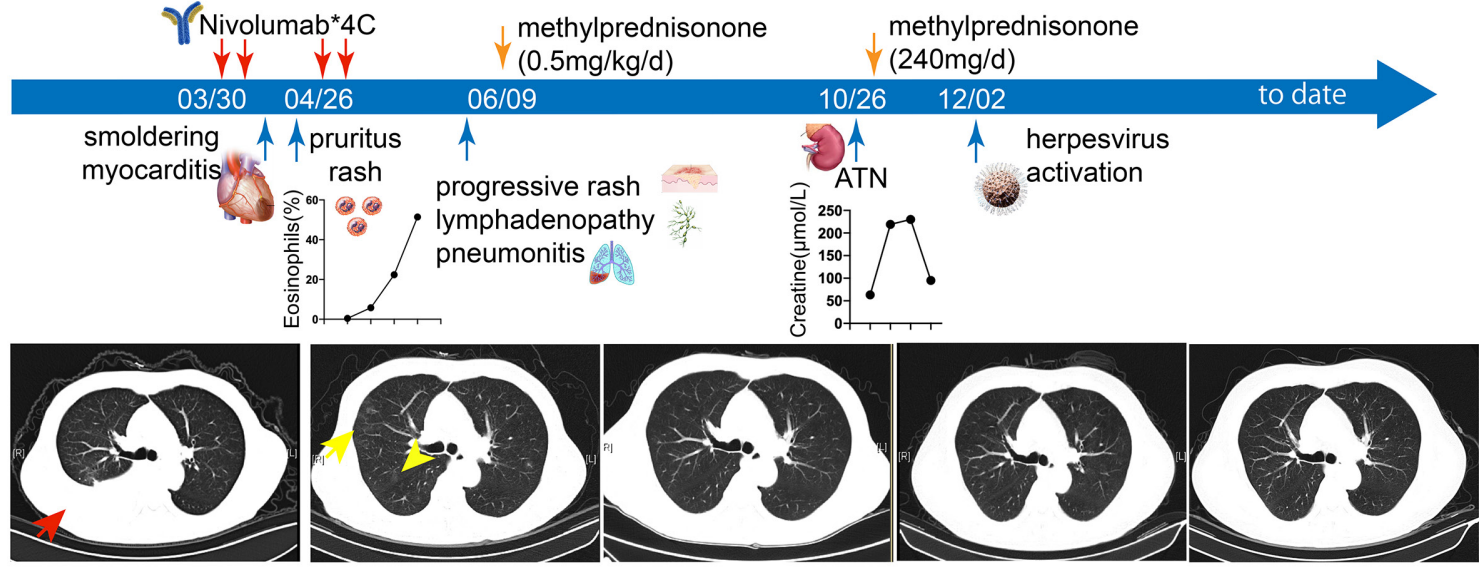

malignant pleural effusion

pneumonitis

recovered

Figure 1 Clinical course and outcome of the patient. The top panel shows the patient's representative abdominal tumor lesion during and after treatment with nivolumab (red arrows indicate lesions). The middle panel shows the timeline of clinical events. The bottom row shows the representative images of thorax CT scan (red arrow indicates malignant pleural effusion and yellow arrows indicate pneumonitis). ATN, acute tubulointerstitial nephritis; CR, complete response; PR, partial response.

January 2019. Pathology of the tumor revealed HER2 staining was negative. Six cycles of adjuvant chemotherapy with oxaliplatin plus S-1 were given postoperatively in July 2019. However, 3 months later, a follow-up contrastenhanced CT scan indicated peritoneal and abdominal metastasis. Then he participated in a clinical trial aimed to compare the efficacy between patients treated with fruquintinib plus paclitaxel and patients treated with paclitaxel alone. In February 2020, the re-examination of CT revealed multiple metastasis in the abdominal and pelvic cavity and abdominal wall, as well as pleural effusion in the right side, indicating progression of disease. The main metastatic lesion was located in the abdominal wall $(8.3 \mathrm{~cm} \times 4.9 \mathrm{~cm}$, figure 1$)$. Thus, he was withdrawn from the clinical trial and discontinued any medication for 2 months. The unblinding of the trial data has not yet been carried out so far. Next, on March 30, 2020, he began to receive a total of four doses of nivolumab $(200 \mathrm{mg})$, every 2 weeks, as the third-line therapy. Three weeks after initiating nivolumab, he had an unexpected scattered myocardial edema and subepicardial late gadolinium enhancement (LGE) on his cardiovascular magnetic resonance (CMR, part of test items in a clinical trial; figure 2). Serum myocardial injury markers and ECG were within normal limit, and he denied any discomfort. A possible smoldering myocarditis was suspected and carefully monitored without interventions. One more week later, he developed a pruritic rash on the back and upper extremities. Prior to this, he had no personal history of skin rashes, autoimmune diseases, or family history of autoimmune disease. Moreover, he denied taking any other drugs except nivolumab in the past 3 months (nivolumab for 1 month). Topical corticosteroid cream and moisturizers as well as oral cetirizine were used, but with little effects. The rash had progressively worsened

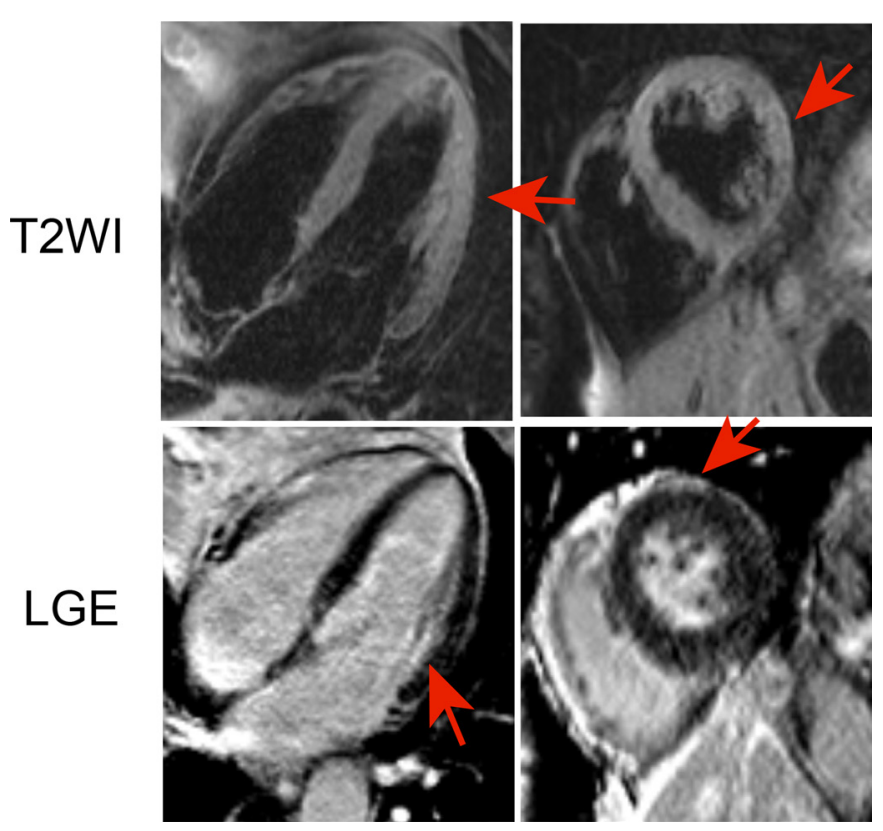

Figure 2 Representative T2WI and LGE pattern. The red arrows indicate myocardial edema at the lateral segment of the left ventricle. Scattered LGE was predominantly distributed at the inferior and lateral segments of the left ventricle with a sub-epicardium pattern. LGE, late gadolinium enhancement; T2WI, T2-weighted image. 
A $\quad 06 / 03 / 2020$ (2.3 months after nivolumab initiation) $B$
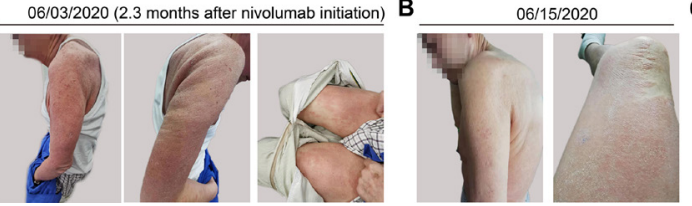

C

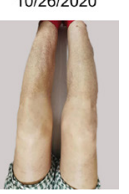

Figure 3 Rash in this patient. (A) Diffuse erythematous eruption with desquamation, drying on chest, back, and extremities of the patient. (B) Partial resolution of eruption after 1 week of steroid therapy. (C) Complete resolution of eruption 5 months later.

over the next weeks. He denied any fever, chills, cough or shortness of breath. Physical examination found diffuse erythematous eruption with desquamation, drying on his chest, back, and extremities, affecting more than $50 \%$ of body surface area (figure 3A). There were no pustules or bullae or mucosal involvement. Multiple bilateral axillary tender lymphadenopathy was palpated. Cardiac, pulmonary, and abdominal examinations were unremarkable.

Examination workup showed a progressive eosinophilia: $0.64 \times 10^{9} / \mathrm{L}(5.8 \%)$ [normal reference range (n.r.) $\left.(0.02-0.52) \times 10^{9} / \mathrm{L}, \quad(0.4 \%-8.0 \%) \quad\right]$ at baseline, $1.45 \times 10^{9} / \mathrm{L}(22.4 \%)$ after the second nivolumab dose, and $4.21 \times 10^{9} / \mathrm{L}(51.4 \%)$ after the fourth dose. IgE was gradually increased from within normal limit to $6165 \mathrm{IU} /$ $\mathrm{mL}$ to $9071 \mathrm{IU} / \mathrm{mL}$ (n.r. $<200 \mathrm{IU} / \mathrm{mL}$ ) during the course. A hypochromic microcytic anemia (about $90 \mathrm{~g} / \mathrm{L}$ ) was detected. Serum iron was low. Erythropoietin (EPO) $(14.8 \mathrm{mIU} / \mathrm{mL}$, n.r. $(4.3-29.0 \mathrm{mIU} / \mathrm{mL}))$ was normal and Coombs test was negative. Hepatic function, renal function, electrolyte, urine routine test, stool routine test, and fecal occult blood test were all within normal limits.
Cancer and insufficiency intake-related anemia was speculated. Thorax CT scan showed multiple patches and nodular hazy shadows suggestive of interstitial pneumonitis in the right lung (figure 1; lower part, yellow arrowheads). The infectious workup was negative. As he denied any other medications, DRESS syndrome secondary to nivolumab was suspected. Nivolumab therapy was permanently discontinued, and oral methylprednisolone (32 $\mathrm{mg}$ /day) was started. His symptoms were relieved gradually (figure 3B,C) and eosinophil count dropped down to normal a month later $\left(0.21 \times 10^{9} / \mathrm{L}, 0.3 \%\right)$. Glucocorticoid was tapered over very slowly to prevent DRESS flares.

Nevertheless, 4 months later, when he was still on oral corticosteroids at $8 \mathrm{mg} /$ day, he reported fatigue. He denied any recent fever, gross hematuria, dysuria, or decreased urine output. Initial workup showed an acute increase in serum creatinine level (from $63 \mu \mathrm{mol} / \mathrm{L}$ progressively elevated to $230 \mu \mathrm{mol} / \mathrm{L}$ (n.r. 44-115 $\mu \mathrm{mol} / \mathrm{L})$ ) over a 2-week period. His renal and ureter ultrasound was also unremarkable. Urine analysis showed no casts or eosinophils but slight hematuria and proteinuria (0.4 g/24 hours). Blood urine nitrogen was normal. Eosinophil count was normal, but IgE was still high (2075 $\mathrm{IU} / \mathrm{mL}$ ). A test for antinuclear antibodies was borderline positive (1:100). Serum protein electrophoresis was negative for monoclonal spike, and flow cytometry for leukemia/lymphoma was negative. Due to the absence of a clear explanation for the acute progressive renal failure, renal biopsy was performed. Histopathological analysis revealed diffuse interstitial edema with an intense, patchy lymphocytic plasma cells and neutrophils infiltration. Ten per cent of renal tubules displayed atrophy (figure 4A).
A

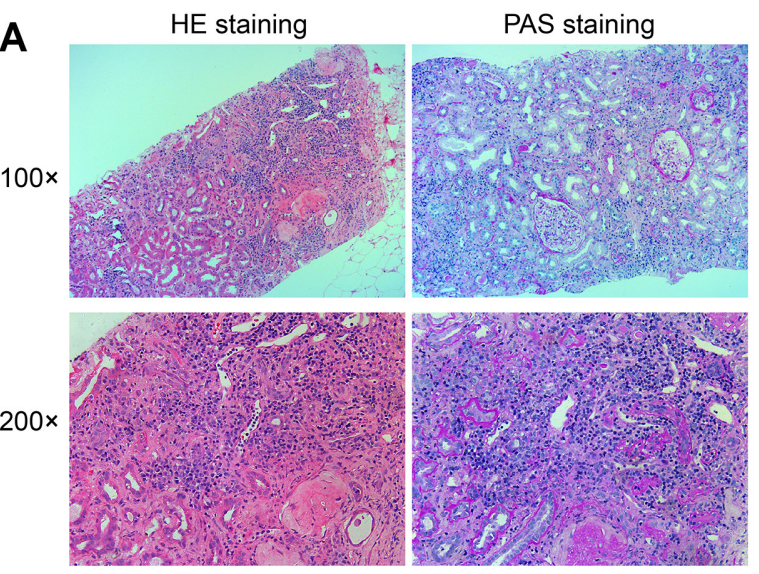

C

PD-L1 IHC staining in primary gastric cancer tissue

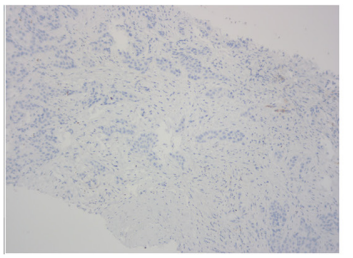

B

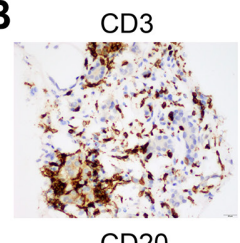

$\mathrm{CD} 20$

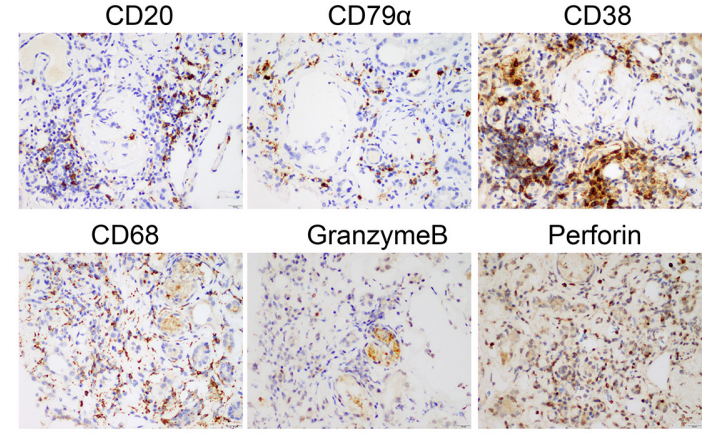

PD-1

PD-L1 1\#

PD-L1 ${ }^{2 \#}$

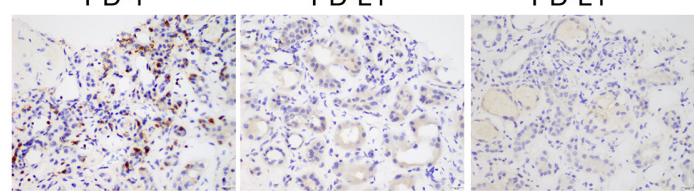

Figure 4 Renal biopsy with severe interstitial inflammation. (A) H\&E and PAS staining of renal biopsy. (B) IHC staining of biomarkers as indicated in renal biopsy. (C) IHC staining of PD-L1 in primary gastric cancer biopsy. IHC, Immunohistochemistry; PAS, periodic acid-Schiff. 
Immunofluorescence showed IgG, IgA, IgM, C3, C4, C1q, kappa, lambda, and fibrin (FIB) were all negative. Severe acute tubulointerstitial nephritis (ATN) was diagnosed. Nivolumab was postulated as the most likely culprit drug, while omeprazole could not be fully ruled out for suspicion. The reasons were as follows: (1) ICIs can induce Acute kidney injury (AKI); in a combined analysis of 3695 patients treated with ICIs, the overall incidence of AKI was $2.2 \%{ }^{14}$; (2) renal involvement was a common component of DRESS; and (3) accumulating evidence concerned AKI after the proton pump inhibitor (PPI) application, ${ }^{15} 16$ and he was also on omeprazole together with steroids for 4 months. It is difficult to discern the postulated causative drugs from the aspect of symptoms and pathology because the symptoms were both unspecific and ATN was the most commonly reported pathology for both renal irAEs ${ }^{17}$ and PPI-associated AKI. ${ }^{1518}$ We were more inclined to consider his AKI as a continuum of nivolumab-induced DRESS. Moreover, because we prescribed him high-dose glucocorticoids (intravenous methylprednisolone 240 $\mathrm{mg}$ /day) immediately after renal biopsy, omeprazole was withheld to prevent gastrointestinal bleeding. Creatinine rapidly normalized (about $95 \mu \mathrm{mol} / \mathrm{L}$ ) in 1-week period, supporting the speculation it was nivolumab rather than omeprazole that was the most probable causative drug inducing ATN. Renal function has remained stable since then. Steroids were tapered over regularly. He developed a sequential herpes zoster virus activation in his left leg 5 weeks later and recovered after support care.

Of note, he only received four doses of nivolumab as the third-line therapy and discontinued any antitumor therapy for the next 12 months, but complete response was notably achieved and sustained (figure 1, upper panel). He still has a good performance without tumor relapse when this case report was revised.

Most of the reporting on irAEs comes from clinical studies and, hence, findings about the mechanisms of irAEs are limited. ${ }^{5}$ We tried to provide mechanistic insights into the pathogenesis of nivolumab-associated DRESS in this patient. We first conducted immunohistochemistry analysis on renal biopsy tissue. As shown in figure $4 \mathrm{~B}$, the infiltrating cells were positive for CD3, CD4, CD8, CD20, CD79 $\alpha$, CD38, and CD68 staining. C4d was negative. Granzyme B and perforin were also positive. These results indicated that the ATN in this patient was mediated by immune attack. PD-L1 expression is regarded as a good predictor for response to ICI therapy. However, PD-L1 expression in renal biopsy, together with the primary gastric cancer tissue, was consistently negative (figure 4C). PD-1 was expressed in $5 \%$ of interstitial cells.

We also isolated peripheral blood mononuclear cells from him at the onset and resolution of ATN. As illustrated in figure 5A, the fraction of $\mathrm{CCR}^{-} \mathrm{CD}^{2} 5 \mathrm{RA}^{+}$effector $\mathrm{CD}^{+}$and $\mathrm{CD}^{+} \mathrm{T}$ cells were strikingly high at ATN onset and decreased at the stage of ATN resolution. When gated on $\mathrm{CD}^{+}$cells, a substantial shift to Th2 response, a significant enrichment of Th17 cells and a depletion of regulatory $\mathrm{T}$ (Treg) cells were observed at the acute injury stage (figure $5 \mathrm{~B}$ ). In parallel, granzyme $\mathrm{B}$, granzyme A and perforin gated on $\mathrm{CD} 8^{+} \mathrm{T}$ cells were highly expressed at ATN onset (figure 5C). Coinhibitory molecules such as PD-1, CTLA-4, and LAG3 were weakly expressed in both $\mathrm{CD}^{+}$and $\mathrm{CD} 8^{+}$cells (figure $5 \mathrm{D}$ ). We further evaluated the concentration of plasma cytokines by using the inflammatory multiplexed panel. Of 14 proinflammatory and regulatory cytokines and chemokines evaluated, interleukin (IL)-17A, IL-17F, interferon gamma (IFN- $\gamma$ ), and IL-5 were increased at ATN onset (figure 5E). This exaggerated immune phenotype was consistent with a type IV hypersensitivity reaction. Moreover, PD-1 pathway inhibition may partly mediate it as well. Expectedly, the aberrantly activated immune response partly returned to balance at ATN resolution.

We also tried to figure out why the immune response to nivolumab was so strong in this patient from the genetic aspect. Next-generation sequencing on his primary gastric tumor tissue and blood cells showed the tumor mutation burden was 6.35 muts/Mb (moderate) in tissue and $<1$ muts $/ \mathrm{Mb}$ (low) in blood. The microsatellite instability (MSI) score was 0, indicating the tumor was microsatellite stable. A somatic EP300 p.Q741* mutation at the frequency of $6.16 \%$ was detected in tumor biopsy. EP300 mutation was reported to be associated with tumor mutation burden (TMB) and promoted antitumor immunity in patients with bladder cancer. ${ }^{19}$ We also found EP300 mutation was correlated with more immune cells infiltration and higher GZMB, GZMA, and GZMH gene expressions in the Cancer Genome Atlas (TCGA) stomach adenocarcinoma datasets by using TIMER V.2.0 analysis tool $^{20}$ (figure 6). These results suggested the somatic EP300 mutation may be related to his good antitumor response.

Finally, we performed validated human leukocyte antigen (HLA) typing assay of this patient's DNA from peripheral blood cells as HLA alleles are well-recognized genetic risk factors for non-ICI-related DRESS. ${ }^{21-23}$ Both classical HLA-I loci of the patient are listed in table 1. Remarkably, HLA-A*31:01, a well-documented DRESS risk allele, ${ }^{13} 22-25$ was indeed found in this patient. This HLA risk loci may render him to be a susceptible individual to DRESS.

\section{DISCUSSION}

DRESS is a severe adverse drug reaction characterized by extensive rash accompanied with multiple visceral organ involvement, eosinophilia, and lymphadenopathy. Though rare, a mortality rate up to $10 \%$ of DRESS syndrome was reported. ${ }^{26}$ Due to the heterogenous nature and a general lack of physician knowledge of this condition, DRESS syndrome is undoubtedly globally underdiagnosed. Currently, two diagnostic criteria have been proposed by the International Registry of Severe Cutaneous Adverse Reactions group and the Japanese Consensus Group: the RegiSCAR criteria and the 
A

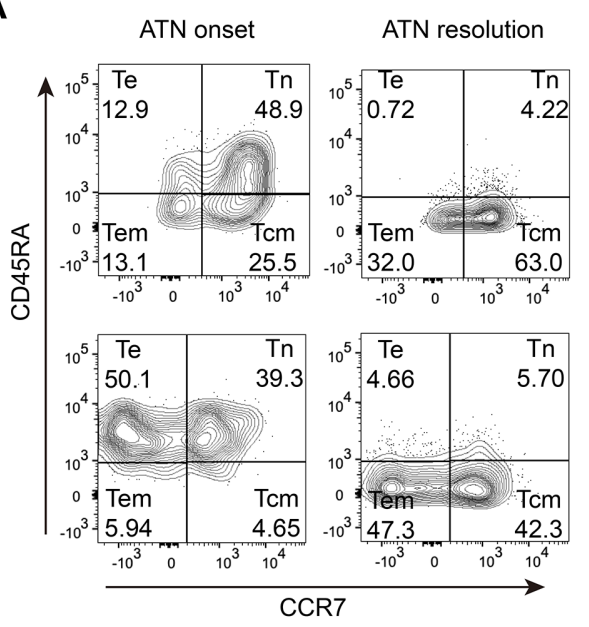

B
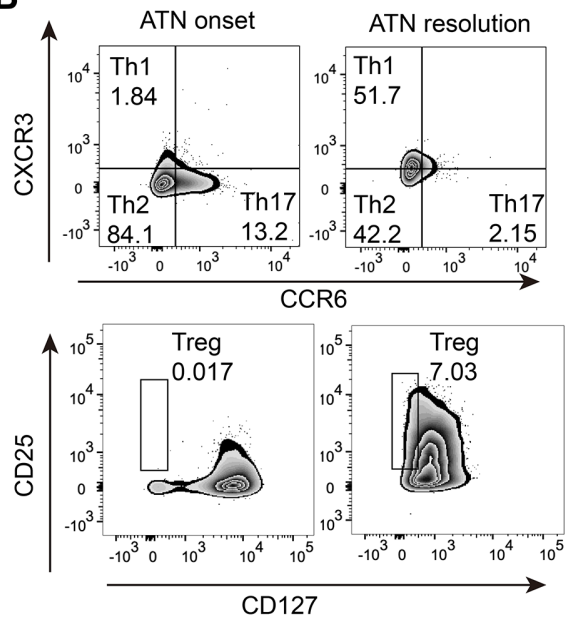

C
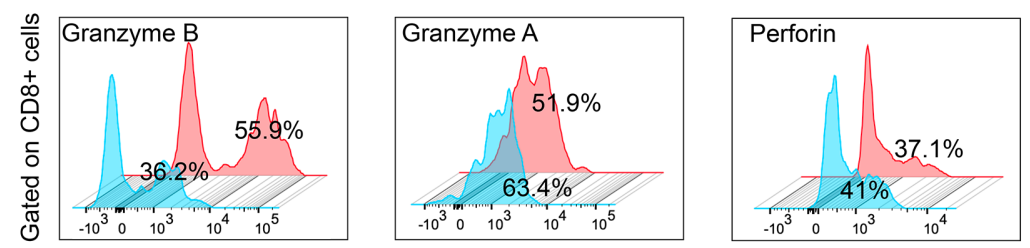

ATN onset

ATN resolution

D

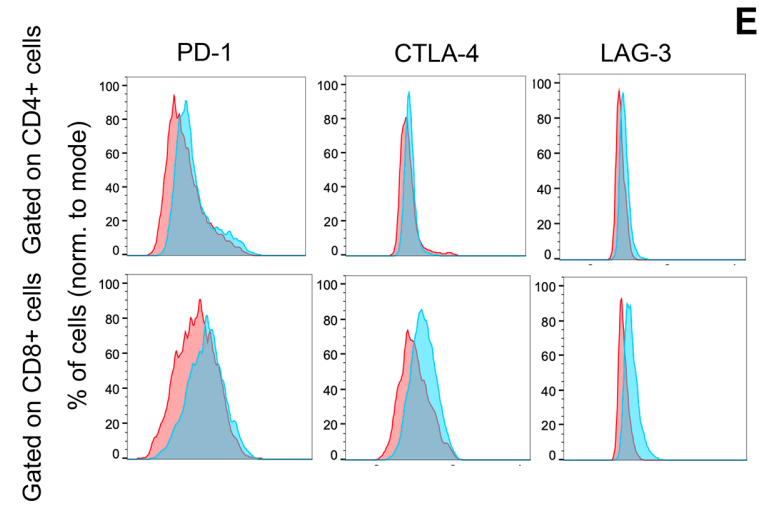

E

\begin{tabular}{lcc}
\hline Cytokines $(\mathrm{n} . \mathrm{r})$. & ATN onset & ATN resolution \\
\hline IL-17A $(\leq 4.74 \mathrm{pg} / \mathrm{ml})$ & 39.789 & 0.656 \\
IL-17F $(\leq 4.66 \mathrm{pg} / \mathrm{ml})$ & 7.593 & 2.606 \\
IL-22 $(\leq 3.64 \mathrm{pg} / \mathrm{ml})$ & 1.384 & 0.638 \\
TNFa $(\leq 4.50 \mathrm{pg} / \mathrm{ml})$ & 3.923 & 0.838 \\
TNF $\beta(\leq 2.54 \mathrm{pg} / \mathrm{ml})$ & 2.671 & 0.001 \\
IFNy $(\leq 4.43 \mathrm{pg} / \mathrm{ml})$ & 8.179 & 15.318 \\
IL-1 $(\leq 3.4 \mathrm{pg} / \mathrm{ml})$ & 0.001 & 0.001 \\
IL-2 $(\leq 6.64 \mathrm{pg} / \mathrm{ml})$ & 5.644 & 1.286 \\
IL-4 $(\leq 4.19 \mathrm{pg} / \mathrm{ml})$ & 3.152 & 0.299 \\
IL-5 $(\leq 4.15 \mathrm{pg} / \mathrm{ml})$ & 12.237 & 0.685 \\
IL-6 $(\leq 11.09 \mathrm{pg} / \mathrm{ml})$ & 3.856 & 28.814 \\
IL-8 $(\leq 15.71 \mathrm{pg} / \mathrm{ml})$ & 14.709 & 9.351 \\
IL-10 $(\leq 4.5 \mathrm{pg} / \mathrm{ml})$ & 5.47 & 2.109 \\
IL-12p70 $(\leq 10.18 \mathrm{pg} / \mathrm{ml})$ & 2.524 & 0.867 \\
\hline
\end{tabular}

Figure 5 Immune status assessment during ATN onset and resolution. (A) T-cell subpopulation distribution. (B) Th1, Th2, Th17, and Treg cell distribution during ATN onset and resolution. (C) Granzyme B, granzyme A, and perforin expression gated on CD8 ${ }^{+}$cells. (D) PD-1, CTLA-4, and LAG-3 expression gated on CD4 ${ }^{+}$and $C D 8^{+}$cells. (E) Cytokine expression during ATN onset and resolution. ATN, acute tubulointerstitial nephritis; IFN- $\gamma$; interferon gamma; IL, interleukin; Tcm, central memory T cell; Te, effector T cell; Tem, effector memory T cell; Tn, naïve T cell; TNF, tumor necrosis factor.

Japanese Consensus Group criteria. ${ }^{13}$ There is no fundamental difference between the two criteria, but the HHV-6 reactivation is not included in the RegiSCAR criteria. Except for the documented fever, our patient presented with typical DRESS clinical pictures: rash, eosinophilia, lymphadenopathy, pulmonary involvement, and stepwise development of renal failure as well as sequential herpesvirus activation. These prolonged clinical symptoms after the initiation of nivolumab substantiates nivolumab as an inciting agent of DRESS. In previous literature, the most frequently reported culprit drugs were aromatic anticonvulsants (carbamazepine and phenobarbital), antibiotic sulfonamides (dapsone and sulfamethoxazole) and allopurinol. ${ }^{1327} 28$ Documented ICIs as offending drugs for DRESS syndrome were rare. To our knowledge, there is one case of nivolumab-associated DRESS, ${ }^{10}$ one case of ipilimumab-related DRESS, ${ }^{11}$ and one case of ipilimumab in combination with nivolumab-associated DRESS $^{12}$ in published literature. Our case is different from the aforementioned cases because (1) this is the first case that showed HLA genotypes may lay the genetic susceptible foundation for ICI-induced DRESS; (2) this case displayed a typically prolonged course with stepwise development of organ damage and sequential herpesvirus activation in ICI-related DRESS; and (3) we had a comprehensive immune status assessment both in blood and renal tissue to better understand the mechanisms. Luckily, all the four ICI-related DRESS cases were responsive to high dosage of steroids therapy, indicating rapid recognition and timely steroid therapy are the cornerstones for DRESS syndrome. 
A

Immune infiltration in TCGA STAD datasets( $\mathrm{N}=439)$
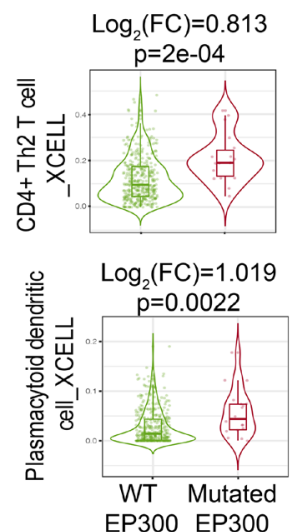

B

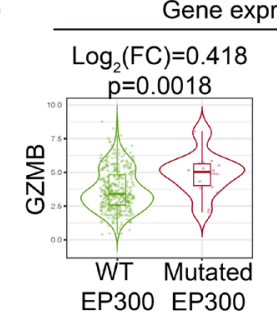

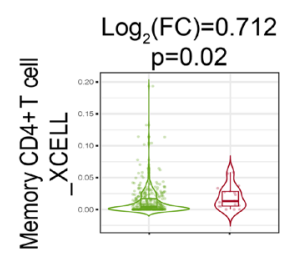

$\log _{2}(F C)=2.142$

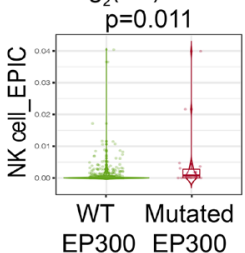

EP300 EP300 $\log _{2}(F C)=0.299$

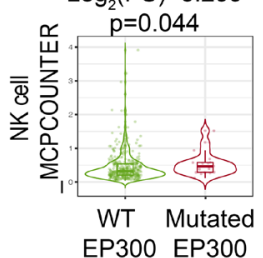

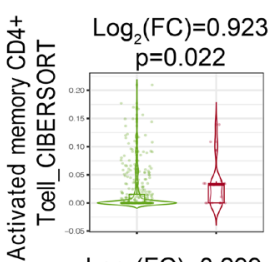

and an intense infiltration of lymphocytes and phagocytes with high expression of perforin in renal biopsy, as well as elevated serum IFN- $\gamma$ and IL-6, were observed at ATN onset, indicating type IVc hypersensitivity reaction was also involved in DRESS. This contention was supported by findings that high numbers of $\mathrm{CD}^{+}$cells and granzyme $\mathrm{B}^{+}$cells were also infiltrated in skin in previous studies. ${ }^{29} 30$ Type IV hypersensitivity reactions were commonly seen in autoimmune diseases. $\mathrm{T}$ cells were the major mediators that cause tissue damage by activation of B lymphocytes and phagocytes, production of cytokines, and direct granzyme/perforin-mediated cytotoxicity. The renal tissue biopsy and blood test in this patient support the concept that type IV hypersensitivity dominated nivolumabinduced DRESS, which we referred to as 'off-target effect'. Drugs such as carbamazepine, allopurinol or sulfamethoxazole have been shown to be directly recognized by the $\mathrm{T}$ cell receptor (TCR) on non-covalent interactions with HLA and/or TCR molecules. Three models, that is, a hapten/prohapten model, a pharmacological interaction model, and an altered peptide model, have been proposed to explain how $\mathrm{T}$ cells were activated by specific drugs. ${ }^{21} 3132$ We proposed the degradation metabolites of nivolumab, such as amino acids or small peptide molecules, were the most likely antigens that stimulate $\mathrm{T}$ cells in our case. It was likely these metabolites form covalent or non-covalent bonds with peptide residues in serum or cellular proteins, thus eliciting the T-cell immune response. Additionally, other antigens released by cell death (tumor or non-tumor) undergoing cytotoxic attack or even nivolumab itself also have the potential to constitute the immunogenicity. Further molecular studies are needed to explore the nature of drug-specific immune responses.

Yet, irAEs are more than just toxicities from a drug; they are a window into immune regulation. Given the broad expression of PD-1 in various immune cell types and its nature as a coinhibitory molecule, it is tempting to speculate that immunopathogenesis of nivolumabinduced DRESS may also result from the aberrantly lower threshold of tolerance to multiple antigens, including tumor-associated antigen as well as autoantigens, particularly taking into consideration the fact that this patient has reached complete tumor regression. We referred to this mechanism as an 'on-target effect'. We speculated both on-target and off-target effects contributed to the ultimate disease phenotype. That is to say, the drug, that is, nivolumab, produced specific antigens and activated specific effector T cells due to 'off-target effects' in susceptible individuals. In parallel, the PD-1 pathway was unfortunately yet intentionally blocked by nivolumab due to its on-target effects. Thus, the immune balance was greatly tipped and an incredibly aberrant immune response was triggered and sustained, which caused tumor regression as well as broad tissue damage. This patient luckily responded well to steroids, but we believe his immune system is still at high risk to be intolerant of many autoantigens. It requires close monitoring in the future. 
One of the most important findings about this patient is that we identified HLA-A*31:01, a specific predictor for carbamazepine-DRESS ${ }^{22-25}$ in this patient. This is the first study indicating HLA profiles may also constitute the genetic risk factor for ICI-induced DRESS. The extremely strong association between HLA-A*31:01 and non-ICIinduced DRESS was observed in multiple populations including Europeans, ${ }^{22}{ }^{25}$ Han Chinese, ${ }^{25}$ Japanese, ${ }^{24}$ and African ${ }^{23}$ populations. The OR reached as high as 57.6. ${ }^{21}$ In addition to HLA-A*31:01, other HLA alleles such as HLA-B*58:01, HLA-B*13:01, and HLA-A*32:01 were also well-established genetic risk factors for non-ICIinduced DRESS. ${ }^{21}$ HLA complex performs the function of presenting peptides to specific T-cell receptors. The epitope number and the peptide affinity for HLA molecules is haplotype specific. The same antigen may display different immunogenicity in individuals with different HLA profiles. ${ }^{33}$ In this regard, different HLA molecules are reasonably able to enhance or repress specific $\mathrm{T}$ cell-mediated or antibody response to specific antigens. Indeed, HLA signature has been tightly correlated to either ICI therapy treatment response ${ }^{34} 35$ or other irAEs, including pneumonitis, ${ }^{33}$ encephalitis ${ }^{36}$ thyroid dysfunctions, ${ }^{37}$ and adrenal insufficiency. ${ }^{38}$ Given the exponential application of ICI therapy, HLA-typing assay may be valuable to screen genetic susceptible or potential beneficial individuals before ICIs treatment.

Another quite interesting finding is that we detected an unexpected myocardial edema and LGE on CMR only after 3 weeks of initial nivolumab therapy, preceding the onset of any symptoms of irAEs. As his serum myocardial injury markers were negative, we speculated he may have possible smoldering myocarditis. CMR is the gold-standard non-invasive imaging test for diagnosis in myocarditis due to its excellent spatial resolution and its ability to provide tissue characterization. ${ }^{39}$ One cohort of 103 patients diagnosed with ICI myocarditis showed that $48 \%$ of the overall cohort presented with LGE on CMR. ${ }^{40}$ For this patient, his myocardium may have already been suffering from immune attack at that early time. In support of our speculation, a study by $\mathrm{Oh}$ et al showed a rapid diversification of both the $\mathrm{CD} 4^{+}$and $\mathrm{CD} 8^{+}$T-cell repertoires and the generation of self-reactive clonotypes within 2 weeks of ipilimumab treatment, preceding onset of irAEs. ${ }^{41}$ CMR may have the potential to be the most sensitive screen tool for immune-related myocarditis.

\section{CONCLUSIONS}

We report the first visceral pathology proven case of nivolumab-related DRESS in a genetic susceptible individual. It showed a typically prolonged course (more than half a year) with stepwise development of renal failure and sequential herpes zoster virus activation after pneumonitis and pruritus rash, which has not been observed in previous few ICI-associated DRESS syndrome. Type IV hypersensitivity-related off-target effect and PD-1 block-related on-target effect may both account for the immunopathogenesis of this emerging condition. A DRESS risk allele HLA-A*31:01 was identified in this patient. Our study helps increase the awareness of clinicians for ICI-mediated DRESS. HLA genotyping may be an appropriate tool to screen genetic susceptible individuals before ICI therapy.

Contributors LA conceived and wrote the study. JG performed the flow cytometry analysis. SZ performed the CT/MRI scan and radiological analysis. QL, Y-HC, QL, and DW treated the patient. YW, XJ, and YJ performed the renal biopsy and pathological evaluation. JL performed the next-generation sequencing and analysis. TL and YY designed or/and supervised this project and revised the study.

Funding This project was supported by grants from the National Natural Science Foundation of China (81800485 and 81802356), Shanghai Rising-Star Program (21QA1401600), Committee of Science and Technology of Shanghai (19DZ1910102), and Clinical Research Fund of Zhongshan Hospital (2020ZSLC39).

Competing interests None declared.

Patient consent for publication Obtained.

Ethics approval This study was approved by the ethics committee of Zhongshan Hospital affiliated to Fudan University. Informed consent was obtained from the patient for submission of the case.

Provenance and peer review Not commissioned; externally peer reviewed.

Open access This is an open access article distributed in accordance with the Creative Commons Attribution Non Commercial (CC BY-NC 4.0) license, which permits others to distribute, remix, adapt, build upon this work non-commercially, and license their derivative works on different terms, provided the original work is properly cited, appropriate credit is given, any changes made indicated, and the use is non-commercial. See http://creativecommons.org/licenses/by-nc/4.0/.

\section{ORCID iDs}

Luoyan Ai http://orcid.org/0000-0002-1093-2018

Duojiao Wu http://orcid.org/0000-0003-2562-8825

\section{REFERENCES}

1 Ai L, Xu A, Xu J. Roles of PD-1/PD-L1 pathway: signaling, cancer, and beyond. Adv Exp Med Biol 2020;1248:33-59.

2 Das S, Johnson DB. Immune-related adverse events and anti-tumor efficacy of immune checkpoint inhibitors. J Immunother Cancer 2019;7:306.

3 Rogado J, Sánchez-Torres JM, Romero-Laorden N, et al. Immunerelated adverse events predict the therapeutic efficacy of anti-PD-1 antibodies in cancer patients. Eur J Cancer 2019;109:21-7.

4 Anderson R, Theron AJ, Rapoport BL. Immunopathogenesis of immune checkpoint Inhibitor-Related adverse events: roles of the intestinal microbiome and Th17 cells. Front Immunol 2019;10:2254.

5 Esfahani K, Elkrief A, Calabrese C, et al. Moving towards personalized treatments of immune-related adverse events. Nat Rev Clin Oncol 2020;17:504-15.

6 Kennedy LB, Salama AKS. A review of cancer immunotherapy toxicity. CA Cancer J Clin 2020;70:86-104.

7 Brahmer JR, Lacchetti C, Schneider BJ, et al. Management of immune-related adverse events in patients treated with immune checkpoint inhibitor therapy: American Society of clinical oncology clinical practice guideline. J Clin Oncol 2018;36:1714-68.

8 Kumar V, Chaudhary N, Garg M, et al. Current diagnosis and management of immune related adverse events (irAEs) induced by immune checkpoint inhibitor therapy. Front Pharmacol 2017;8:49.

9 Naidoo J, Page DB, Li BT, et al. Toxicities of the anti-PD-1 and antiPD-L1 immune checkpoint antibodies. Ann Oncol 2015;26:2375-91.

10 Lu J, Thuraisingam T, Chergui M, et al. Nivolumab-associated dress syndrome: a case report. JAAD Case Rep 2019;5:216-8.

11 Voskens CJ, Goldinger SM, Loquai C, et al. The price of tumor control: an analysis of rare side effects of anti-CTLA-4 therapy in metastatic melanoma from the ipilimumab network. PLoS One 2013;8:e53745.

12 Mirza S, Hill Ebone', Ludlow SP, et al. Checkpoint inhibitorassociated drug reaction with eosinophilia and systemic symptom syndrome. Melanoma Res 2017;27:271-3.

13 Shiohara T, Mizukawa Y. Drug-induced hypersensitivity syndrome (DiHS)/drug reaction with eosinophilia and systemic symptoms (DRESS): An update in 2019. Allergol Int 2019;68:301-8. 
14 Cortazar FB, Marrone KA, Troxell ML, et al. Clinicopathological features of acute kidney injury associated with immune checkpoint inhibitors. Kidney Int 2016;90:638-47.

15 Nochaiwong S, Ruengorn C, Awiphan R, et al. The association between proton pump inhibitor use and the risk of adverse kidney outcomes: a systematic review and meta-analysis. Nephrol Dial Transplant 2018;33:331-42.

16 Chen G, Ning L-J, Qin Y, et al. Acute kidney injury following the use of different proton pump inhibitor regimens: a real-world analysis of post-marketing surveillance data. J Gastroenterol Hepatol 2021;36:156-62.

17 Wanchoo R, Karam S, Uppal NN, et al. Adverse renal effects of immune checkpoint inhibitors: a narrative review. Am J Nephrol 2017;45:160-9.

18 Al-Aly Z, Maddukuri G, Xie Y. Proton pump inhibitors and the kidney: implications of current evidence for clinical practice and when and how to deprescribe. Am J Kidney Dis 2020;75:497-507.

19 Zhu G, Pei L, Li Y, et al. Ep300 mutation is associated with tumor mutation burden and promotes antitumor immunity in bladder cancer patients. Aging 2020;12:2132-41.

$20 \mathrm{Li} \mathrm{T}, \mathrm{Fu}$ J, Zeng Z, et al. TIMER2.0 for analysis of tumor-infiltrating immune cells. Nucleic Acids Res 2020;48:W509-14.

21 Bellón T. Mechanisms of severe cutaneous adverse reactions: recent advances. Drug Saf 2019;42:973-92.

22 McCormack M, Alfirevic A, Bourgeois S, et al. HLA-A*3101 and carbamazepine-induced hypersensitivity reactions in Europeans. $N$ Engl J Med 2011;364:1134-43.

23 Ksouda K, Affes H, Mahfoudh N, et al. HLA-A*31:01 and carbamazepine-induced dress syndrom in a sample of North African population. Seizure 2017;53:42-6.

24 Mushiroda T, Takahashi Y, Onuma T, et al. Association of HLA-A*31:01 screening with the incidence of carbamazepineinduced cutaneous adverse reactions in a Japanese population. JAMA Neurol 2018;75:842-9.

25 Genin E, Chen D-P, Hung S-I, et al. HLA-A*31:01 and different types of carbamazepine-induced severe cutaneous adverse reactions: an international study and meta-analysis. Pharmacogenomics $J$ 2014;14:281-8.

26 Cacoub P, Musette P, Descamps V, et al. The dress syndrome: a literature review. Am J Med 2011;124:588-97.

27 James J, Sammour YM, Virata AR, et al. Drug reaction with eosinophilia and systemic symptoms (dress) syndrome secondary to furosemide: case report and review of literature. Am J Case Rep 2018;19:163-70.

28 Kardaun SH, Sekula P, Valeyrie-Allanore L, et al. Drug reaction with eosinophilia and systemic symptoms (dress): an original multisystem adverse drug reaction. results from the prospective RegiSCAR study. Br J Dermatol 2013;169:1071-80.
29 Ortonne N, Valeyrie-Allanore L, Bastuji-Garin S, et al. Histopathology of drug rash with eosinophilia and systemic symptoms syndrome: a morphological and phenotypical study. Br J Dermatol 2015;173:50-8.

30 Cho Y-T, Liau J-Y, Chang C-Y, et al. Co-existence of histopathological features is characteristic in drug reaction with eosinophilia and systemic symptoms and correlates with high grades of cutaneous abnormalities. J Eur Acad Dermatol Venereol 2016;30:2077-84.

31 Pichler WJ, Adam J, Watkins S, et al. Drug hypersensitivity: how drugs stimulate $T$ cells via pharmacological interaction with immune receptors. Int Arch Allergy Immunol 2015;168:13-24.

32 Illing PT, Mifsud NA, Purcell AW. Allotype specific interactions of drugs and HLA molecules in hypersensitivity reactions. Curr Opin Immunol 2016;42:31-40.

33 Correale P, Saladino RE, Giannarelli D, et al. HLA expression correlates to the risk of immune checkpoint inhibitor-induced pneumonitis. Cells 2020;9. doi:10.3390/cells9091964. [Epub ahead of print: 25 Aug 2020].

34 Chowell D, Morris LGT, Grigg CM, et al. Patient HLA class I genotype influences cancer response to checkpoint blockade immunotherapy. Science 2018;359:582-7.

35 Correale P, Saladino RE, Giannarelli D, et al. Distinctive germline expression of class I human leukocyte antigen (HLA) alleles and Drb1 heterozygosis predict the outcome of patients with non-small cell lung cancer receiving PD-1/PD-L1 immune checkpoint blockade. J Immunother Cancer 2020;8:e000733.

36 Chang $\mathrm{H}$, Shin $\mathrm{Y}-\mathrm{W}$, Keam B, et al. Hla-B27 association of autoimmune encephalitis induced by PD-L1 inhibitor. Ann Clin Trans/ Neurol 2020;7:2243-50.

37 Inaba $\mathrm{H}$, Ariyasu $\mathrm{H}$, Iwakura $\mathrm{H}$, et al. Distinct clinical features and prognosis between persistent and temporary thyroid dysfunctions by immune-checkpoint inhibitors. Endocr J 2021;68:231-41.

38 Yano S, Ashida K, Sakamoto R, et al. Human leucocyte antigen DR15, a possible predictive marker for immune checkpoint inhibitor-induced secondary adrenal insufficiency. Eur J Cancer 2020;130:198-203.

39 Biesbroek PS, Hirsch A, Zweerink A, et al. Additional diagnostic value of CMR to the European Society of cardiology (ESC) position statement criteria in a large clinical population of patients with suspected myocarditis. Eur Heart J Cardiovasc Imaging 2018;19:1397-407.

40 Zhang L, Awadalla M, Mahmood SS, et al. Cardiovascular magnetic resonance in immune checkpoint inhibitor-associated myocarditis. Eur Heart J 2020;41:1733-43.

41 Oh DY, Cham J, Zhang L, et al. Immune toxicities elicted by CTLA-4 blockade in cancer patients are associated with early diversification of the T-cell repertoire. Cancer Res 2017;77:1322-30. 